\title{
A New Method for the Parametric Identification of DC Machines Using MATLAB Identification Toolbox and Experimental Measurements
}

\author{
Boudjemai Hamza ${ }^{1}$, Ardjoun Sid Ahmed El Mehdi1 ${ }^{1, *}$, Chafouk Houcine ${ }^{2}$ and Denai Mouloud ${ }^{3}$ \\ ${ }^{1}$ IRECOM Laboratory, Faculty of Electrical Engineering, Djillali Liabes University, Sidi Bel-Abbes, Algeria \\ ${ }^{2}$ Normandy University, UNIROUEN, ESIGELEC, IRSEEM, 76000 Rouen, France \\ ${ }^{3}$ School of Engineering and Computer Science, University of Hertfordshire, Hatfield, United Kingdom
}

\begin{abstract}
This paper proposes a new approach for the identification of a DC machine (DCM) parameters to build a mathematical model considering different dynamic regimes, which characterize the operation of the studied machine. The proposed solution is simple and is based on the combination of classical identification methods and those available in the identification toolbox of MATLAB. The results obtained experimentally are significantly better and clearly show that the proposed approach is simple to implement and the DCM model is obtained quickly with a reasonable accuracy.
\end{abstract}

Keywords. DC machine, Parameter identification, Matlab Ident Toolbox, dSPACE 1104.

\section{Introduction}

Nowadays, there are several real systems from different disciplines such as mechanics, electronics, thermal, chemistry, physical, etc. around us. The study, analysis, monitoring, and control of these systems, require as a first step a reasonably accurate mathematical model. Identification is the process by which a simple model structure is derived from a complex system. Experimental measurements consisting of input and output data are recorded from the system at an appropriate sampling period and used in the identification process.

For the identification of the DCM parameters, most researchers use classical methods because of their simplicity. However, these methods require a lot of testing on the machine which increases the risk to damage the actual system and they do not give best results, especially when using them to determine the specific inductances ( $L e, L a)$ and the mechanical parameters $(f, J)$. Thus, their performance remains insufficient for complex systems which are nonlinear and exhibit time-varying dynamics [1]. In [2], the authors present three simple techniques for the identification of DCM parameters based only on the direct measurement of current and speed. The advantages and disadvantages of each method are presented. Other researchers have studied the digital identifiers methods to reduce the number of tests on the machine and to ensure rapid detection of parameters change in real time and under variable environmental conditions (variation in load, machine overheating, short-circuit fault, etc.). Among these works, the recursive least squares method [3], constrained optimization technique [4], Kalman filter [5], identification of nested loop systems [6], artificial neurons networks [7], Tabu research technique [8], adaptive Tabu search technique [9] and genetic algorithms [10-11]. It should be noted that all these methods have many advantages for estimation DCM settings. However most of these methods remain difficult to implement and require a large memory for processing information in real time.

Several software tools are currently available (MATLAB, LabView, LTspice, etc.) and can be interfaced to real-world systems via powerful digital signal processing (DSP) and digital microcontroller (dsPIC), dSPACE, etc.). Several identification tools can be implemented to find a mathematical model (continuous time or discrete time) of a real system. Among these identification tools we can cite: the toolbox, developed for MATLAB, named CONTSID (Continuous-Time System Identification). The use of this toolkit is described in [12-14]. Thus, we find the estimation graphical interface and "MATLAB Ident toolbox" complete analysis. With the availability of all these means, we have decided to search for a new solution for identifying DCM parameters based on classic identification methods (measurement of the rotor resistance and the constant torque) and the identification methods available in MATLAB software (Ident toolbox).

This paper is structured as follows: a more general description of the DCM model is presented in section 2 . Section 3 summarizes the main classical identification methods used to calculate the DCM parameters. A detailed description of the proposed identification approach for the estimation of the DCM parameters is

* Corresponding author: elmehdi.ardjoun@univ-sba.dz 
described in Section 4. Section 5 presents the experimental setup used to validate the estimated models. Finally, the results obtained and the conclusions are summarized in Sections 6 and 7 respectively.

\section{Description of the DCM model}

The modeling of DCM is based on the following simplifying assumptions: The saturation effect and the skin effect are neglected. Eddy currents and the phenomenon hysteresis are also neglected. The inductors and resistors are constant.

The DCM used in this study is rated $0.1 \mathrm{~kW}, 220 \mathrm{~V}$, $2000 \mathrm{rpm}$. The operation of this machine can be modeled by the following system of equation:

$$
\left\{\begin{array}{l}
u_{e}=R_{e} i_{e}(t)+L_{e} \frac{d i_{e}(t)}{d t} \\
u_{a}=R_{a} i_{a}(t)+L_{a} \frac{d i_{a}(t)}{d t}+e(t) \\
J \frac{d \Omega(t)}{d t}=C_{e m}(t)-C_{r}(t)-f \Omega(t)
\end{array}\right.
$$

With:

$C_{e m}(t)=k i_{a}(t), e(t)=k \quad(t), k=k_{m},(=$ constant $)$

Where $u_{e}$ is the excitation (field) voltage, $u_{a}$ the armature voltage, $i_{e}$ excitation current, $i_{a}$ armature current, $R_{e}, L_{e}$ resistance and inductance of the excitation circuit (field), $R_{a}, L_{a}$ armature resistance and inductance, $f$ coefficient of friction, $J$ is the inertia, is the mechanical speed, $C_{e m}$ electromagnetic torque, $C_{r}$ resistive torque, $k_{m}$ the constant geometric of the DCM, flux of excitation, $k$ is the torque constant or back-emf constant.

Applying the Laplace transform to the above equations leads to the following transfer function:

$$
H(s)=\frac{\Omega(s)}{U_{a}(s)}=\frac{\frac{k}{k^{2}+R_{a} f}}{\frac{L_{a} J}{k^{2}+R_{a} f} s^{2}+\frac{L_{a} f+R_{a} J}{k^{2}+R_{a} f} s+1}
$$

Finally, to determine the model of the DCM, it is necessary to find experimentally the following parameters: $R_{a}, L_{a}, k, f$ and $J$.

\section{Identification based on classical methods}

\subsection{Determination of rotor resistance}

Several methods are available for measuring the rotor resistance. Some of these methods are summarized below:

\subsubsection{Ohmmeter method}

This method is based on the use of an ohmmeter. However, the value of the rotor resistance depends on the position of the slippery contacts with respect to the collector. Therefore, we need to perform several measurements for different positions of the slippery contacts as shown in Table 1. But it should be noted that these measurements are recorded at the operating temperature.

Table 1. Measurement of rotor resistance by the ohmmeter method

\begin{tabular}{|c|r|c|}
\hline Position & Ra (ohm) & \multirow{2}{*}{ Average value } \\
\hline 1 & 53.11 & \multirow{1}{*}{} \\
\hline 2 & 53.90 & \\
\hline 3 & 52.40 & \multirow{2}{*}{ R $_{\text {amoy }}=54.13 \mathrm{ohm}$} \\
\hline 4 & 54.13 \\
\hline 5 & 56.00 & \\
\hline 6 & 54.60 & \\
\hline 7 & 53.90 & \\
\hline 8 & 56.40 \\
\hline 9 & 52.70 & \\
\hline 10 & 53.80 & \\
\hline 11 & 54.50 & \\
\hline
\end{tabular}

\subsubsection{Voltmeter - ammeter method}

In this method, the tests are carried out continuously and with locked rotor $\left(=0, \mathrm{e}=0\right.$ and $\left.\mathrm{di}_{\mathrm{a}} / \mathrm{dt}=0\right)$. The machine is operated at rated current to avoid excessive heating and the measurements are recorded at reduced voltages. The resistance value can be determined by the following relation:

$$
R_{a}=\frac{U_{a}}{I_{a}}
$$

The results obtained are presented in the following table:

Table 2. Measurement of rotor resistance by the voltmeter-ammeter

\begin{tabular}{|c|c|c|c|}
\hline $\mathbf{U a}(\mathbf{V})$ & $\mathbf{I a}(\mathbf{A})$ & $\mathbf{R a}(\mathbf{o h m})$ & \multirow{2}{*}{ Average value } \\
\hline 10 & 0.19 & 52.63 & \\
\hline 13 & 0.25 & 52.00 & \\
\hline 15 & 0.28 & 53.57 & \multirow{2}{*}{ R $_{\text {amoy }}=52.8 \mathrm{ohm}$} \\
\hline 20 & 0.38 & 52.63 & \\
\hline 24 & 0.45 & 53.33 & \\
\hline 27 & 0.52 & 51.92 & \\
\hline 30 & 0.56 & 53.57 & \\
\hline
\end{tabular}

Note: The two methods used for measuring rotor resistance give very close values, which show that we are not far from the real value of the rotor resistance. But as it is known from the operating principle of an ohmmeter: it measures the voltage across the resistor by injecting a very low DC current compared to the actual current flowing through the rotor conductors, which leads to very high values of the resistance. Because of this, we have decided to use the value of the resistance measured by the voltmeter-ammeter method in the remaining calculations. 


\subsection{Determination of rotor inductance}

Among the most widely used methods for measuring the rotor inductance, we can mention the following:

\subsubsection{Index testing method}

In this method, the tests are carried out with locked rotor $(=0, \mathrm{e}=0)$. So equation (2) becomes:

$$
u_{a}=R_{a} i_{a}(t)+L_{a} \frac{d i_{a}(t)}{d t}
$$

So:

$$
i_{a}(t)=\frac{U_{a}}{R_{a}}\left(1-e^{\frac{-t}{\tau_{e}}}\right)
$$

With:

$$
\tau_{e}=\frac{L_{a}}{R_{a}}
$$

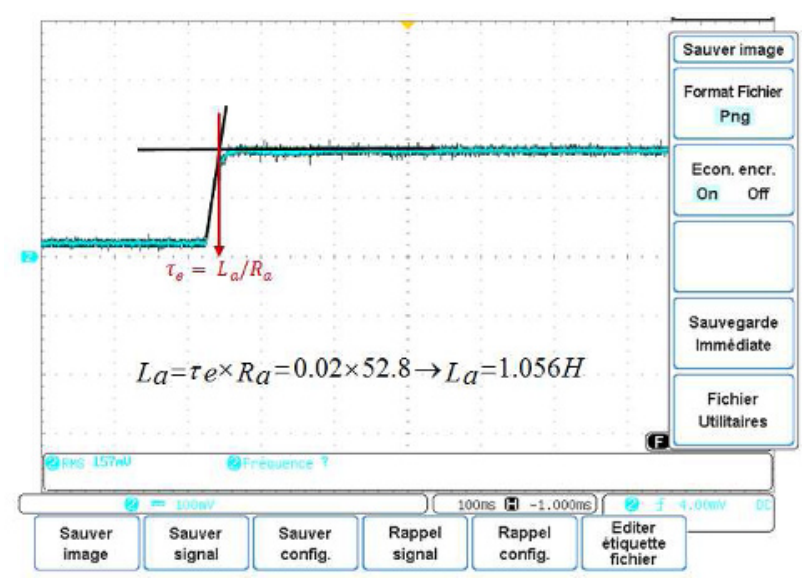

Fig. 1. Locked rotor current response.

\subsubsection{AC current measurement}

To perform this measurement, the rotor of the motor is supplied with an alternating voltage with an open inductor. The expression of the inductance is given by:

$$
L_{a}=\frac{1}{\omega} \sqrt{\left(\frac{U_{a}}{I_{a}}\right)^{2}-R_{a}^{2}}
$$

With: $\quad=2 f, f=50 H z$.

Table 3. Determination of rotor inductance

\begin{tabular}{|c|c|c|c|}
\hline $\mathbf{U a}(\mathbf{V})$ & Ia (A) & La (H) & Average value \\
\hline 10 & 0.0496 & 0.62 & \multirow{2}{*}{ Lamoy $_{\text {am }}=0.74 \mathrm{H}$} \\
\hline 20 & 0.0905 & 0.68 & \\
\hline 30 & 0.1030 & 0.91 & \\
\hline
\end{tabular}

Note: The two methods used for measuring the rotor inductance give different values. Therefore, one cannot conclude which is the best value. Hence, the average of these two values of the inductance was used ( $L a=0.898$ $H)$.

\subsection{Determination of the constant $k$}

The constant $k$ is defined as the ratio between rotational speed and voltage measurement at no-load by a generator test, therefore:

$$
k=\frac{E}{\Omega}
$$

The results obtained are presented in the following table:

Table 4. Determination of the torque constant or back-emf constant

\begin{tabular}{|c|c|c|c|c|}
\hline $\mathbf{U a}(\mathbf{V})$ & $(\mathbf{r a d} / \mathbf{s})$ & $\mathbf{E}(\mathbf{V})$ & $\mathbf{k}$ & \multirow{2}{*}{ Average value } \\
\hline 050 & 44.390 & 40.060 & 0.90 & \multirow{1}{*}{} \\
\hline 100 & 97.000 & 86.330 & 0.89 & \\
\hline 130 & 130.00 & 116.00 & 0.89 & \multirow{2}{*}{$k_{\text {moy }}=0.891$} \\
\hline 150 & 151.97 & 132.21 & 0.87 & \\
\hline 180 & 191.00 & 171.90 & 0.90 & \multirow{2}{*}{} \\
\hline 200 & 214.00 & 192.90 & 0.90 & \\
\hline 220 & 237.47 & 211.34 & 0.89 & \\
\hline
\end{tabular}

\subsection{Determination of friction coefficient}

At no load, the electromagnetic torque is written in the form next:

$$
C_{e m}=C_{c}+f \Omega
$$

Where: $C_{c}$ constant torque and $f$ friction torque.

To determine $f$, the speed of the machine is varied and the necessary $C_{e m}$ torque values are recorded to plot the curve $C_{e m}=f(\quad)$. The results obtained are presented in the following table:

Table 5. Different measurement to determine the coefficient of friction

\begin{tabular}{|c|c|c|c|}
\hline Ua (V) & Ia (A) & $(\mathbf{r a d} / \mathbf{s})$ & Cem $(N . m)$ \\
\hline 050 & 0.054 & 51.30 & 0.048 \\
\hline 100 & 0.064 & 106.2 & 0.057 \\
\hline 150 & 0.070 & 162.0 & 0.062 \\
\hline 180 & 0.074 & 195.2 & 0.066 \\
\hline 200 & 0.076 & 218.4 & 0.068 \\
\hline 220 & 0.078 & 242.1 & 0.069 \\
\hline
\end{tabular}

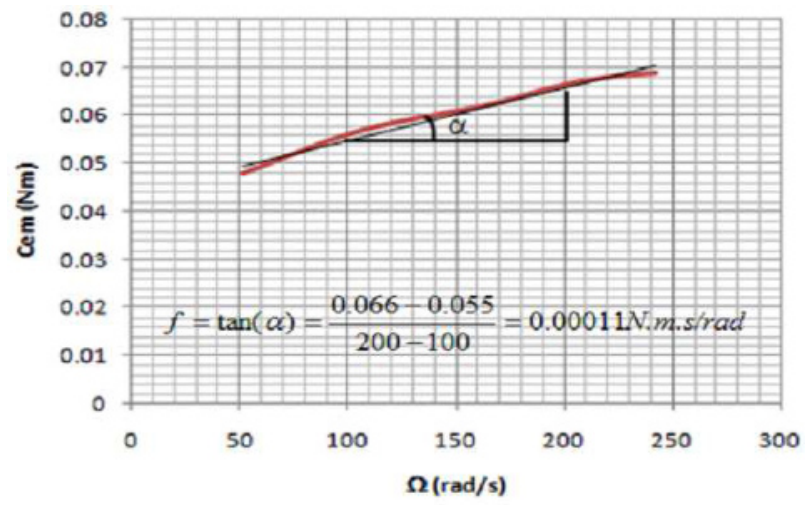

Fig. 2. Electromagnetic torque response as a function of mechanical speed. 


\subsection{Determination of the inertia}

To determine the inertia of the DCM, a low-speed test is performed. After the DCM has reached its rated speed, the supply circuit is disconnected, and we measure the time it takes for the DCM to come to a stop.

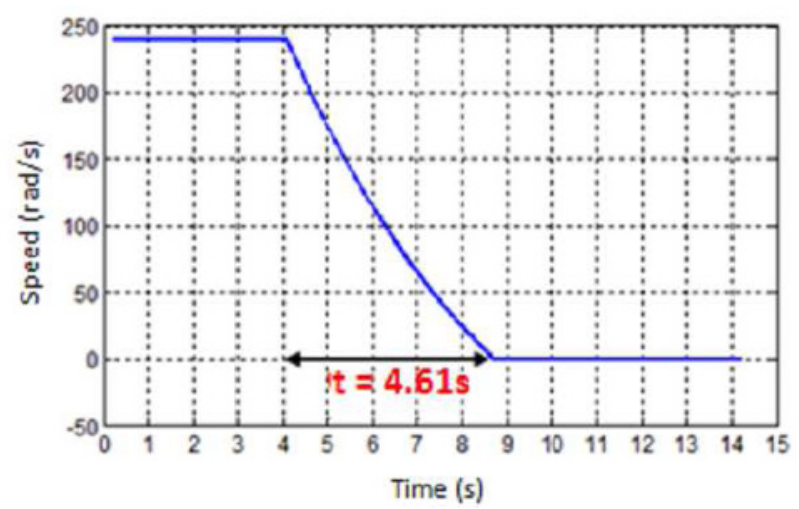

Fig. 3. Mechanical speed response for a deceleration test.

From the deceleration test:

With:

$$
\Omega(t)=\Omega_{0} e^{\frac{-t}{\tau_{m}}}
$$

$$
\tau_{m}=\frac{J}{f}
$$

For $\Omega(t)=\frac{\Omega_{0}}{2}$ we find:

$$
\frac{\Omega_{0}}{2}=\Omega_{0} e^{\frac{-t}{\tau_{m}}} \rightarrow \tau_{m}=\frac{t}{\ln 2}=\frac{4.61}{\ln 2}=6.65 \mathrm{sec}
$$

So:

$J=f \times \tau_{m}=0.00011 \times 6.65=0.0007353 \mathrm{~kg} . \mathrm{m}^{2}$

Finally, for various measurements carried out, we have chosen the following parameters:

$R a=52.8 \mathrm{ohm}, \mathrm{La}=0.898 \mathrm{H}, k=0.891, f=0.00011$ N.m.s $/ \mathrm{rad}, J=0.0007353 \mathrm{~kg} . \mathrm{m}^{2}$.

In this part we have detailed the main classical identification methods needed to calculate DCM parameters. We have seen that the identification of electrical parameters (rotor resistance and the torque constant) by these methods remains simple and easy to implement. On the other hand, for the specific inductances and the mechanical parameters $(f$ and $J)$ we did not find any accurate methods for the same parameter, since we obtained different values. So, to simplify the study and get closer to the real model of the DCM we have used the identification toolbox available in MATLAB software. This study will be the objective of the next section.

\section{New approach to identification of the DCM}

parametric

The approach allows the calculation of all the DCM parameters using the following steps:

- Determination of the electrical parameters (rotor resistance and the torque constant) by classical methods (see section 3).

- Determination of the DCM transfer function using MATLAB Identification toolbox.

The MATLAB Identification toolbox has the graphical user interface (GUI), presented in Fig. 4. This interface offers several types of models (transfer function, state-space representation, polynomial approximations...etc.). But to carry out the identification using this toolbox, it is necessary to fix the order of the model which is not known in advance and which remains a major problem in the identification of all physical systems. In our case, the DCM can be approximated by a second-order transfer function. So thanks to the Identification toolbox, we can easily find the transfer function that models the behavior of the DCM (Fig. 5).

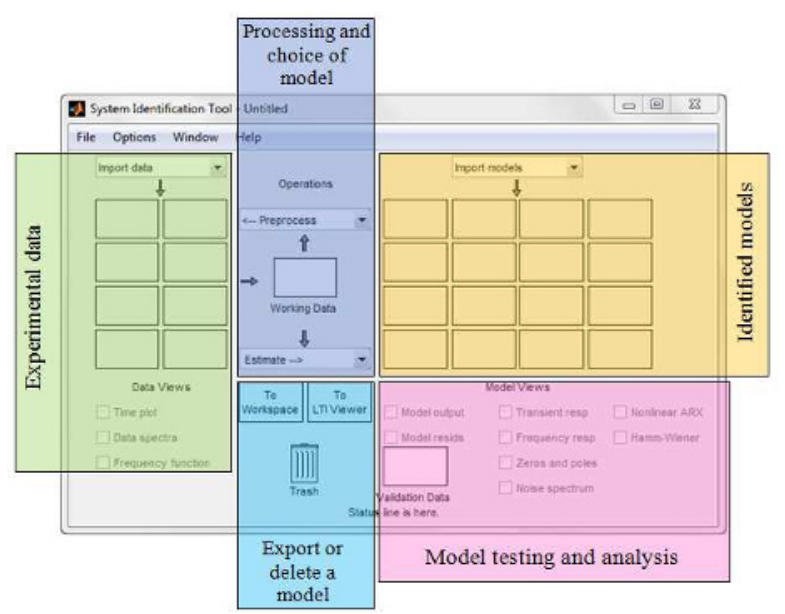

Fig. 4. Description of the command graphical interface Ident from MATLAB.

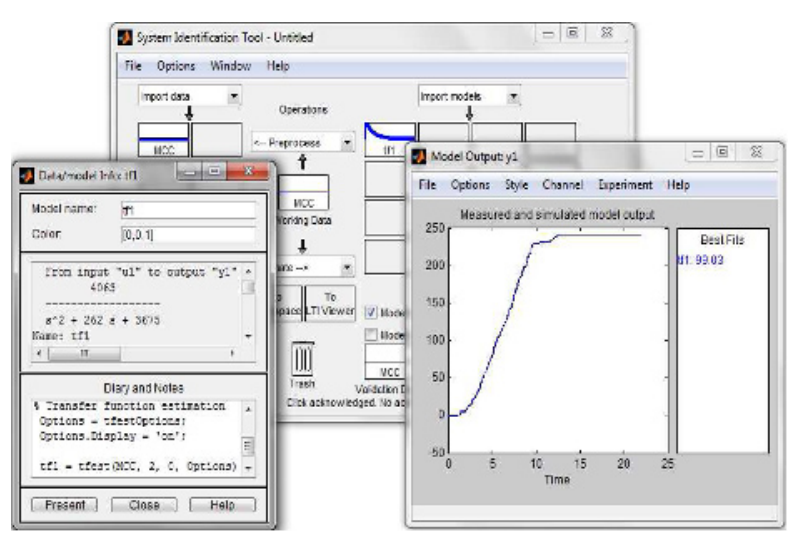

Fig. 5. Identification based on the Ident toolbox for MATLAB. 
- Determination of the DCM Parameters

We have:

The transfer function obtained by the differential equations of the DCM.

$$
H(S)=\frac{\frac{k}{k^{2}+R_{a} f}}{\frac{L_{a} J}{k^{2}+R_{a} f} s^{2}+\frac{L_{a} f+R_{a} J}{k^{2}+R_{a} f} s+1}
$$

The transfer function obtained by the Ident toolbox

$$
H(S)=\frac{1.1056}{\frac{1}{3675} s^{2}+0.0713 s+1}
$$

By comparing the two transfer functions, the following DCM parameters are obtained:

$R a=52.8 \mathrm{ohm}, \mathrm{La}=0.2 \mathrm{H}, k=0.891, f=0.0002276$ N.m.s/rad, $J=0.0011 \mathrm{~kg} . \mathrm{m}^{2}$.

\section{Description of the experimental platform}

The testing platform developed in this paper to validate the DCM models, is described by Figs. 6 and 7. It allows the analysis of the DCM mechanical behavior in real time. In other words, we can constantly compare the actual responses from measurement sensors with the estimated responses at the models output. This comparison is performed using a dSPACE 1104 board. The model is presented at Fig. 7. Thus, a measurement interface composed of current, voltage and speed sensors, provide all readings necessary for the experimental validation of the DCM model.

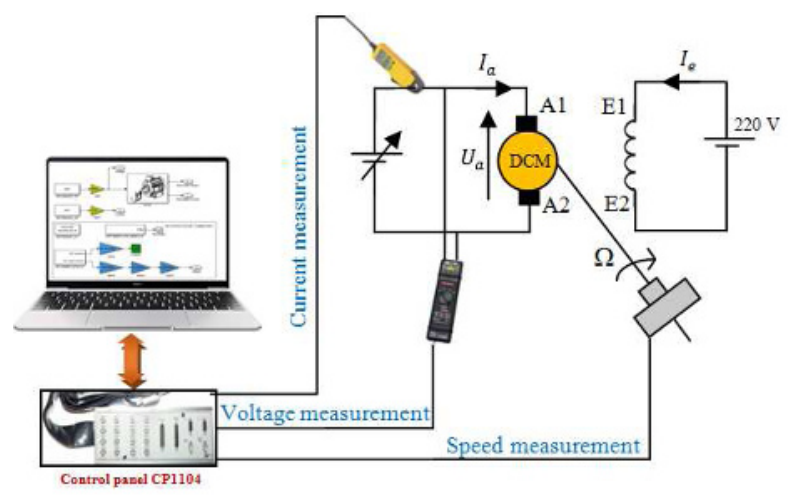

Fig. 6. Schematic diagram of the experimental platform.
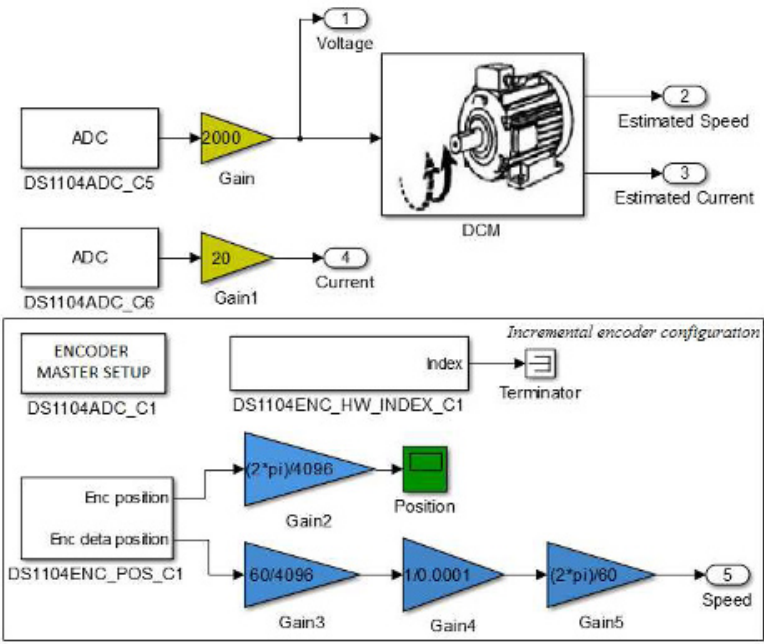

Fig. 7. Simulink / dsPACE model developed to validate the DCM models.

\section{Experimental results}

To test the models of the DCM obtained, we have carried out a no-load test. The measurements obtained are stored in a MATLAB file of type (.mat) thanks to the map dSPACE. The results obtained are presented in Figs. 8-10.

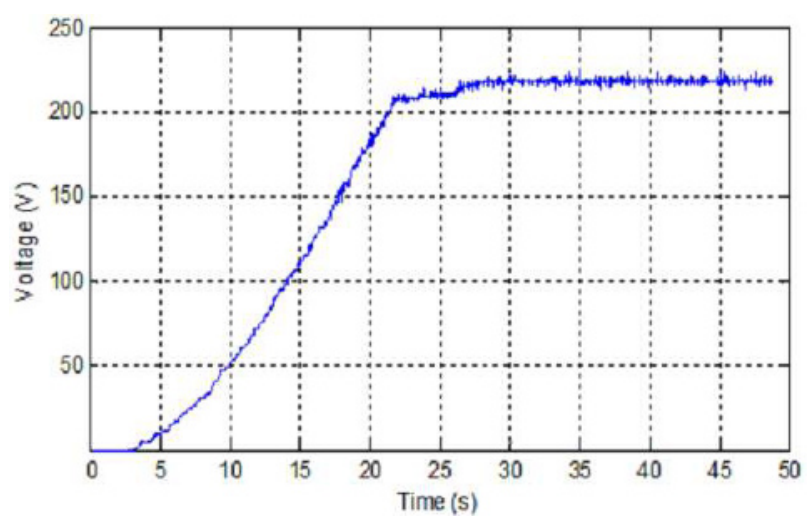

Fig. 8. Rotor voltage curve as a function of time.

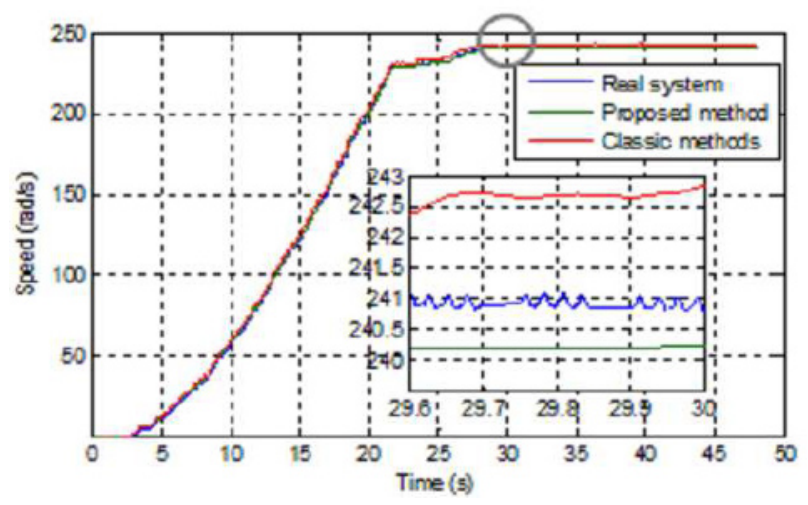

Fig. 9. Comparison between the measured speed and the estimated speeds of the direct current machine. 


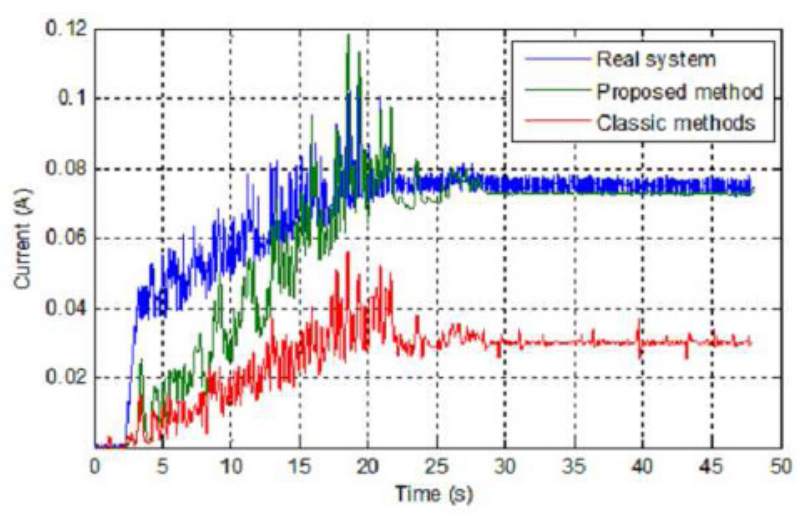

Fig. 10. Comparison between the measured current and the estimated currents of the direct current machine.

From Figs. 9 and 10, we can say that the proposed identification approach allows obtaining a model that is very close to the real DCM. The estimated speed and currents waveforms are very close to the measured ones with a good correlation coefficient of the order (99\%) between the DCM real system and the estimated model.

\section{Conclusion}

In this paper, we have studied the contribution of techniques identifications such as classical methods and identification methods available in the software MATLAB (toolbox Ident), applied to the identification of DCM parameters. Then an experimental platform based on the dsPACE 1104 board was used to validate the models obtained. The results clearly show that the proposed approach offers several advantages for the identification of the DCM parameters. Another benefit is the risk minimization of damaging the actual system and saving time (a low number of tests on the machine). In addition, this solution remains simple, it is accurate and easy to implement (we can always get models very close to the real model). Indeed, this parametric identification method can be an interesting tool to the design of controllers (get an idea of the values initials of the parameters of the correctors used for the DCM control).

In perspective, from an experimental point of view, the dSPACE card remains very expensive. It would be therefore wise to replace it by an affordable microcontroller such as Arduino. On the other hand, for future works, we would like to develop a new algorithm to determine all the DCM parameters, by measuring only the voltage, the current and the speed of the studied machine. Thus, the proposed algorithm can also be used to detect the rapid parameters variations overtime which can be useful to discover machine anomalies, such as heating and short circuits in the DCM stator and /or rotor windings.

\section{References}

1. M. Zegrari, A. Badri et B. Oukarfi, "Identification par la méthode du modèle des paramètres d'une machine à courant continu," IEEE $3^{\text {rd }}$ International Conference: Sciences of Electronic,
Technologies of Information and Télécommunications March 2731, TUNISIA (2005).

2. B. Shanmuga nithya, A. Mythile, S. Pavithra, N. Nivetha, "Parameter identification of a DC motor," International Journal of Scientific \& Technology Research volume9, Issue 02, February (2020).

3. k. Radojka, A. Sanja, and S. Danilo, "Recursive least squares method in parameters identification of DC motors models," SER.: ELEC. ENERG. vol. 18, no. 3, 467-478, December (2005).

4. S. Adewusi, "Modeling and parameter identification of a DC motor using constraint optimization technique," IOSR Journal of Mechanical and Civil Engineering (IOSR-JMCE), Vol. 13, PP 46-56 (2016).

5. S. S. Saab and R. A. Kaed-Bey, "Parameter Identification of a DC Motor: An Experimental Approach," IEEE International Conf. on Elec.Circuit and Systems. (ICECS), vol.4, pp.981-984, (2001).

6. B. Arnaud, "Contributions à l'identification paramétrique de modèles à temps continu : extensions de la méthode à erreur de sortie et développement d'une approche spécifique aux systèmes à boucles imbriquées," Thèse de doctorat, Institut National Polytechnique de Toulouse (INP Toulouse), Octobre (2010).

7. S. Weerasooriya and M. A. El-Sharkawi, "Identification and Control of a DC Motor using Back-Propagation Neural Networks," IEEE Tran. On Engergy Conversion, vol.6, no.4, pp.663-669, (1991).

8. S. Pothiya, S. Chanposri, S. Kamsawang and W. Kinares, "Parameter Identification of a DC Motor Using Tabu Search," KKU Engineering Journal, vol. 30, no.3, pp.173-188, (2003).

9. S. Udomsuk, K-L. Areerak, K-N. Areerak and A. Srikaew, "Parameters Identification of Separately Excited DC Motor using Adaptive Tabu Search Technique,"international conference on advances in energy engineering. IEEE, p. 48-51, (2010).

10. M. Lankarany and A. Rezazade, "Parameter Estimation Optimization Based on Genetic Algorithm Applied to DC Motor," IEEE International Conf. on Electrical Engineering. (ICEE), pp.1-6, (2007).

11. A. Dupuis, M. Ghribi and A. Kaddouri, "Multiobjective Genetic Estimation of DC Motor Parameters and Load Torque," IEEE International Conf. on Ind. Tech. (ICIT), pp.1511-1514, (2004).

12. H. Garnier, M. Mensler et A. Richard, "Continuous-time model identification from sampled data, implementation issues and performance evaluation," International Journal of Control, 76(13):1337-1357, (2003).

13. H. Garnier, M. Gilson, T. Bastogne et H. Zbali, "Contsid : un outil logiciel pour l'identification de modèles paramétriques à temps continu à partir de données expérimentales," In Journées Identification et Modélisation Expérimentale, (2006).

14. H. Garnier, M. Gilson et V. Laurain , "The contsid toolbox for matlab : extensions and latest developments," In 15th IFAC Symposium on System Identification, SYSID, vol. 15 de System Identification, pages 735-740, Saint-Malo,France (2009). 